\title{
Short-term Load Combination Forecasting Based on Evidential Theory Combines with Ant Colony Algorithm-Neural Network
}

\author{
Jing Hua ${ }^{1, \mathrm{a}}$, $\mathrm{Li} \mathrm{Ai}^{1, \mathrm{~b}}$ and Jiatang Cheng ${ }^{1, \mathrm{c}}$ \\ ${ }^{1}$ The Engineering College of Honghe University, Yunnan Mengzi 661199 \\ a46951833@qq.com, ${ }^{\text {b3 } 364508229 @ q q . c o m, ~}{ }^{\circ 578242565 @ q q . c o m ~}$
}

\begin{abstract}
Keywords: Evidence theory; Ant colony algorithm; Neural network; Load forecasting; Combination forecasting
\end{abstract}

\begin{abstract}
In order to improve the accuracy of short-term load forecasting, a combination prediction method is applied of evidence theory combines with ant colony algorithm-neural network. According to a city's actual load data, the ant colony algorithm-neural network as single mode1 is used to its initial forecast. Then the BP and RBF neural network are selected to get the credibility of each model, with forecasting errors and environmental influence. And the evidence theory was employed to fuse them to obtain the combination weight, so short-term load forecast was fulfilled. Examples show that the method by evidence theory to determine the best combination of weight, thus fitting error is small, and with high prediction accuracy. The combination method is suitable for short-term load forecasting prediction and has a certain application value.
\end{abstract}

\section{Introduction}

Power load forecasting is an important task in dispatch department and electricity production, its essence is to build mathematical models according to historical data and describe its development law.

There are many factors which influence the result of power load forecasting, such as economic factors, political factors, weather factors, seasonal factors, geographical factors, the ultimate loads are countless single load superposition of these factors together, and it is difficult to variety factors Affecting quantified, and therefore can not establish an accurate model to describe it.

Some documents apply neural network literature [1], gray system theory [2], genetic algorithm [3], ant colony algorithm [4] in load forecasting and arrives some success.

However, due to differences different forecasting methods, using the single forecasting method, the assumptions and scope have their limitations, which can not always maintain high accuracy. Therefore, it is very necessary to adopt a combination forecasting method which is universal, and can integrate many kinds of forecasting methods. Combination forecasting method is based on establishes different prediction models to predict and the results of a certain weighted sum after the final forecast value. It can make full use of multi-source information, but how to determine the weight of the combination is the difficulty. At present, the average combination forecasting method is simple, but the accuracy of each prediction model is higher. Variance covariance combination forecasting method is used to determine the value of the variance of the historical error, but only in the case of the smaller correlation of each forecast method, the effect is better [5], limiting the scope of its application. The combination forecasting method of neural network can be used as the final prediction result, but there are over fitting problems, which leads to the decrease of the network generalization ability [6].

The evidence theory is a non accurate inference algorithm, it can integrate the expert or data source knowledge or data effectively, it has stronger decision-making ability, it is used to determine the weights of combination forecasting can avoid artificial subjective factors. Therefore, the paper preliminary forecast uses 3 different ants colony neural network model as a single model of short-term load forecasting first, ,and obtains the credibility of the forecast point. Then, using BP, RBF neural network to predict the credibility, after converting it and as evidence body, according to 
the combination rules of evidence theory to combination, in order to obtain the combined model weights, realizes the combination of load forecasting finally.

According to the selected sample data, put it into three independent ant colony neural network model (using the basic ant colony algorithm which different of two parameters [7-8] and BP neural network which used MMAS [9-10] to optimize, called AS-BP1, AS-BP2, MMAS-BP) to deal and get the prediction error of each model, Then, put it and influence factors of external environment as the input of the neural network and the credibility is used as the output of the target, so that the credibility of each single model can be obtained. The schematic diagram of the system is as follows.

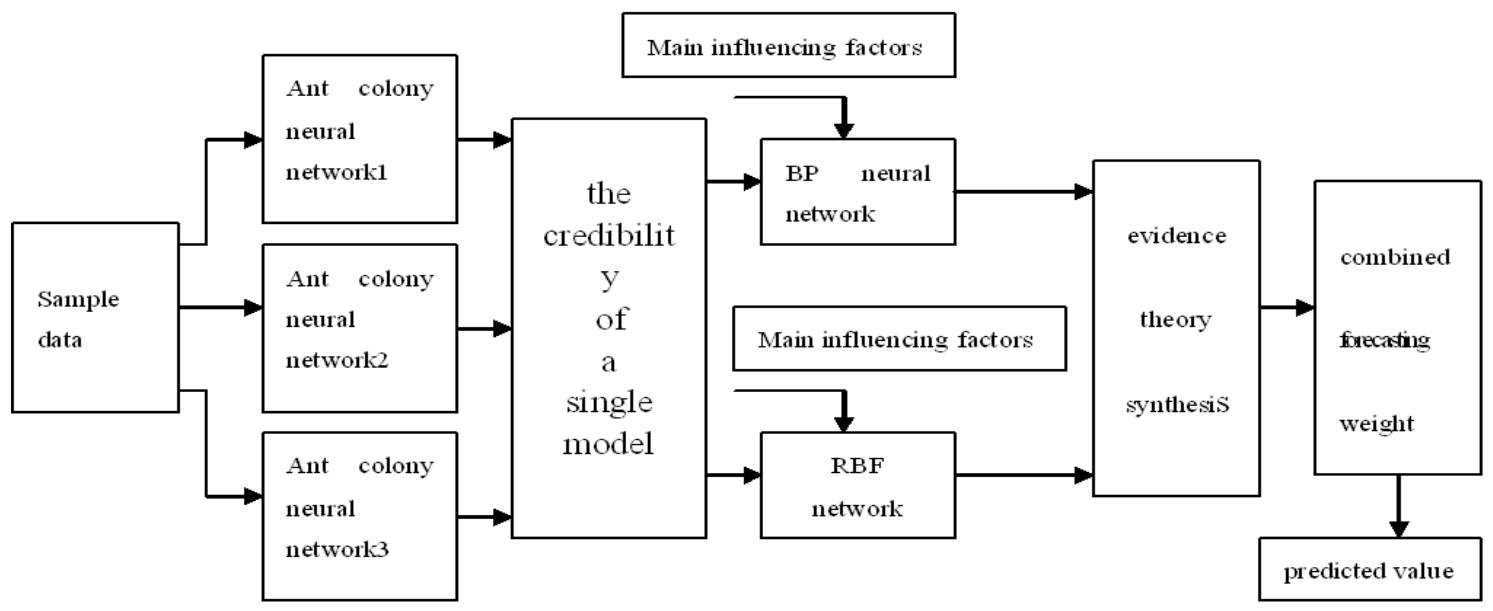

Figure.1 Combined forecasting schematic

\section{Realization of Load Combination Forecasting}

Ant Colony Neural Network. The effect of combined model prediction is influenced significantly by the selection of combined weights and the accuracy of single model prediction. Therefore, the ant colony neural network is chosen as a single prediction model.

The basic idea of the ant colony neural network: a BP neural network has $m$ parameters to be optimized (including all the weights and thresholds), these parameters are sorted, recorded as $p_{i}(1 \leq i \leq m)$. For each parameter $p_{i}$, set it to a smaller random nonzero form set. The ant colony search of food, each ant starting from the ant nets from the collection of $I_{p i}$, travel all of collection, according to the information content and transfer probability of each element ,choose an element from collection of $I_{p 1}$, until the ant reached all food source, then we choose a set of network parameters. Then, the amount of information is updated according to certain rules. However, in the basic ant colony algorithm, it can improve the quality and convergence speed of the solution. But this kind of search will cause premature convergence and premature convergence. In order to improve the performance of the algorithm, Stutzle et al. Proposed the MMAS algorithm. Compared with the basic ant colony algorithm, the MMAS algorithm is improved in the following three points:

(1)Initialization information $\tau_{i j}(t)=c$ is set to the maximum value, which is conducive to the initial stage of the algorithm to search for more solutions;

(2)Only let the ants update the pheromone which to find the optimal solution in the path, in order to find the optimal solution;

(3)Put $\tau_{i j}(t)$ will be limited to $\left[\tau_{\min }, \tau_{\max }\right]$, avoid search stagnation.

Credibility Training. In spite of the neural network, the ant colony neural network model can achieve higher prediction accuracy, but there is a certain error. According to the relative error $e_{i}$ to 
determine the reliability of each ant colony neural network model at a certain point. Reliability $A_{i}$ is expressed as

$$
A_{i}=\left(1 /\left(\left|e_{i}\right|+0.01\right)\right) /\left(\sum_{i=1}^{n}\left(1 /\left(\left|e_{i}\right|+0.01\right)\right)\right)
$$

Among the formula , $\mathrm{n}$ is the number of adapted neural network models; among number of neural network models used for the ant colony, In order to eliminate the extreme cases existence of $e_{i}=0$, the absolute value has increased by a constant 0.01 .

According to the calculated reliability $A_{i}$, take it as the output and the characteristics factors which influencing load change (maximum temperature, minimum temperature, weather conditions and week type) as input, using BP network and RBF network training to obtain the value of credibility. By converting the two models' predictive credibility of the sum is equal to 1 , and as evidence of the theory of evidence, to carry out the integration of evidence.

Evidence Theory. Evidence theory builds a non empty identification framework theory on $\Theta$, the $\Theta$ is consist of a series of mutually exclusive and exhaustive. For any proposition $A$, all belong to a power set $2^{\Theta}$. In the $2^{\Theta}$, if the function $m: 2^{\Theta} \rightarrow[0,1]$ : and to meet

$$
m(\phi)=0, \quad \sum_{A \subset \Theta} m(A)=1
$$

$m$ is the basic belief assignment function the identification framework of $\Theta$. If $A$ is a subset of $\Theta$, and $m(A)>0$, it is called the focal element of the evidence, the collective of all the focal elements is called the nucleus.

If $\mathrm{Bel}_{1}$ and $\mathrm{Bel}_{2}$ are the trust functions on the same recognition framework of, $m_{1}$ and $m_{2}$ respectively is corresponding to the basic trust allocation function, $A_{1}, A_{2}, \cdots, A_{m}$ and $B_{1}, B_{2}, \cdots, B_{n}$ are the focal elements have the following synthesis rules:

$$
m(C)= \begin{cases}\frac{\sum_{A_{i} \cap B=C_{j}} m_{1}\left(A_{i}\right) \times m_{2}\left(B_{j}\right)}{1-k} & C \neq \phi \\ 0 & C=\phi\end{cases}
$$

In the equation, $k=\sum_{A_{i} \cap B=\phi_{j}} m_{1}\left(A_{i}\right) m_{2}\left(B_{j}\right)<1 \quad(i=1,2, \cdots, m ; j=1,2, \cdots, n)$ express the level of conflict between the evidence. When $k \neq 1, m(C)$ has a certain probability assignment; otherwise, it is considered $m_{1}$ and $m_{2}$ contradictory, can not be combined with them. The combination of multiple evidence assembles one to one each other.

Final Prediction. Using the trained ant colony neural network model to predict short-term power load preliminary, and then weighted sum of initial load of combined weight which calculated by evidence theory, you can get the final value of load forecasting.

\section{Application Example of Combination Forecasting}

Use daily maximum load, maximum temperature, minimum temperature, weather conditions and types of week of historical data of a city in 7-8 2013, to verify the feasibility and validity of the method. Here, the weather conditions are divided into four kinds of situations: sunny, cloudy, overcast and rainy, respectively, corresponding to 1,2,3,4, mapping value. For one day, when the weather changes, the weather map is greater as the standard, such as the day the weather is fine with occasional clouds, the weather is that the mapping of the value of 2, and so on. For the week type, Monday to Friday, the value is 1, Saturday, Sunday, the value is 2, and all the data were normalized. According to the ant colony neural network modeling method introduced previously, forecast the preliminary load, and by the historical prediction results, use the evidence theory to automatically 
choose the best combination of weights. Use a week of historical data to verify the analysis as following.

In the initial prediction of a single model, the parameters of the 3 ant colony neural network are set as follows: the network structure 4-10-1, the learning rate is 0.1 , the training target is 0.00001 . Pheromone volatile coefficient $\rho=0.7$, the number of ants $h=10$, the pheromone intensity $Q=0.1$, the maximum number of optimization is 20 , in which the objective function of the MMAS-BP curve is shown in fig 2. After obtained the prediction errors of 3 single models, the reliability function of each model is calculated by the formula (1), and the corresponding weight coefficients are calculated by combination rule formula (3) of the evidence theory. Taking the calculation of the weight of the load forecast in July 16th as an example, the value of AS-BP1 is calculated by the formula (3).

$$
w_{1}=0.2927 \times 0.3108 /(0.2927 \times 0.3108+0.3415 \times 0.2991+0.3658 \times 0.3901)=0.4643
$$

The weights of other single models are similar to that of the calculation, and the fusion results of the theory of evidence are shown in Table 1.

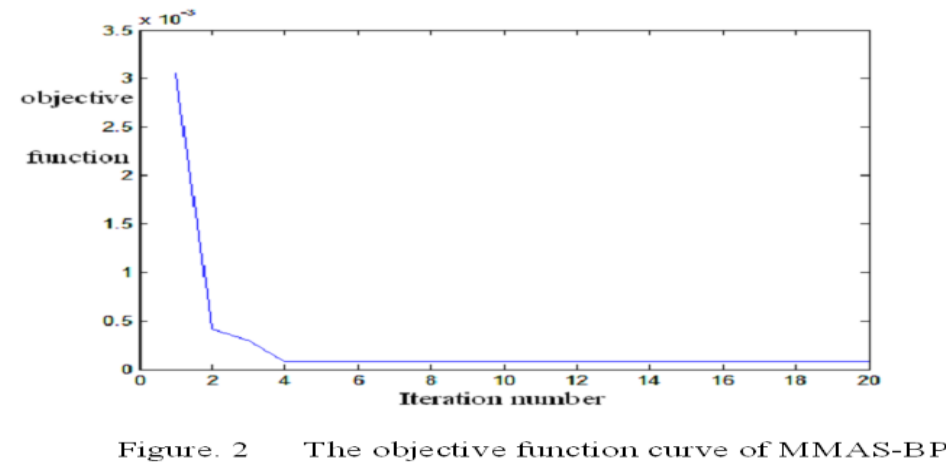

Table 1 the fusion of credibility

\begin{tabular}{|c|c|c|c|c|}
\hline \multirow{2}{*}{$\begin{array}{l}\text { Single } \\
\text { model }\end{array}$} & \multirow[t]{2}{*}{ prediction } & \multicolumn{2}{|c|}{ Evidence Body } & \multirow{2}{*}{$\begin{array}{l}\text { Synthesis } \\
\text { result }\end{array}$} \\
\hline & & $\mathrm{BP}$ & RBF & \\
\hline AS-BP1 & & 0.2927 & 0.3108 & 0.2709 \\
\hline AS-BP2 & & 0.3415 & 0.2991 & 0.3042 \\
\hline MMAS-BP & & 0.3658 & 0.3901 & 0.4249 \\
\hline
\end{tabular}

We can see from Table 1, there are some differences in the prediction results of the credibility of different evidence, but they all think that MMAS-BP is the most reliable. In addition, by using the synthesis rules of evidence theory, the result of the synthesis is the weight of a single model, and the final prediction value can be got after the weighted average.

In order to verify the validity of the method, select the historical data to check the prediction accuracy, and compared with the prediction model of independent ant colony neural network, weighted average combination forecasting method, BP neural network prediction method. The results are shown in Table 2. When using the neural network combination forecast, the forecast value of the independent model is used as the input of the network, and the actual load value is the output. In addition, taking into account the prediction error of a single model in a prediction point may be larger, smooth processing of input data, and that is to say take the average prediction of independent model as input parameters of neural network. 
Table 2 the comparison of different forecasting methods

\begin{tabular}{|c|c|c|c|c|c|c|c|}
\hline \multirow[t]{2}{*}{ number } & \multirow[t]{2}{*}{ Actual value $[\mathrm{MW}]$} & \multicolumn{3}{|c|}{ Single model predictive value } & \multicolumn{2}{|c|}{ Combined model predictive value } & \multirow[b]{2}{*}{$\begin{array}{l}\text { Equal weight } \\
\text { average } \\
\text { combination }\end{array}$} \\
\hline & & AS-BP1 & AS-BP2 & $\begin{array}{l}\text { MMAS-B } \\
\mathrm{P}\end{array}$ & $\begin{array}{c}\text { Evidence theory } \\
\text { combination }\end{array}$ & $\begin{array}{c}\text { Neural network } \\
\text { combination }\end{array}$ & \\
\hline 1 & 26.7 & 25.712 & 27.474 & 27.261 & 26.728 & 27.141 & 26.816 \\
\hline 2 & 26.7 & 25.819 & 26.033 & 26.193 & 26.040 & 26.197 & 26.015 \\
\hline 3 & 24.6 & 25.190 & 23.837 & 23.813 & 24.417 & 24.293 & 24.280 \\
\hline 4 & 25.7 & 24.184 & 24.569 & 24.734 & 24.843 & 24.707 & 24.496 \\
\hline 5 & 32.5 & 30.485 & 33.963 & 31.623 & 31.892 & 32.176 & 32.024 \\
\hline 6 & 34.3 & 35.672 & 32.654 & 35.192 & 34.179 & 34.619 & 34.506 \\
\hline 7 & 34.3 & 33.443 & 34.986 & 34.917 & 34.550 & 34.462 & 34.449 \\
\hline
\end{tabular}

As can be seen from Fig. 2, in the seventh and second samples, the evidence theory combination forecasting error is greater than the results of a single prediction model, this is because the combination of prediction model is greatly affected by a single prediction. But from the whole, according to the order of Tab 2, the mean square errors of the 3 methods were $22.86 \%, 26.93 \%$, $33.23 \%$, respectively. At the same time, the absolute error based on evidence theory combination forecasting method fluctuation range is small, the output of the model is good in stability, and the actual value has better consistency, indicating that the method is feasible for the prediction of short-term power load.

\section{Conclusions}

Put the ant colony algorithm into BP neural network training, as a single short-term power load forecasting model, overcome the defects of conventional BP algorithm has slow convergence and easy to fall into the local minimum, the prediction precision is improved. And based on the prediction error of the single prediction model to determine its credibility, then get the weight of the combination forecast, finally realize the power load forecasting. The example shows that the method has high prediction accuracy and can provide a way of thinking for other complex systems by means of the equal weight average method and the neural network combination forecasting method.

\section{Acknowledgements}

Science Research Foundation of Yunnan Provincial Department of Education（2015Y453）

\section{References}

[1] Ning Lu, Jian-zhong Zhou .Application of particle swarm optimization neural network model in short term load forecasting [J]. Power system protection and control, 2010, 38(12): 65-68.

[2] Jia-ke Li, Ya-jiao Li etc. Multi variable grey neural network model for non point source pollution load forecasting $[\mathrm{J}]$. Journal of Northwest Agriculture and Forestry University (NATURAL SCIENCE EDITION) 2011, 29(3): 229-234.

[3] Yong Zhang, Yun Yang, Application of improved GA-BPN in short term power load forecasting [J]. Computer engineering and Applications, 2009, 45(13):223-226.

[4] Jing Wang, Bo Liu, Yan-hong Feng. Application of ant colony neural network in short term load forecasting [J]. 2008, 29(7):1797-1799, 1837. 
[5] Min Zeng, Yi Feng, Da Liu. Multi model combined electricity price forecasting based on evidence theory [J]. Chinese Journal of Electrical Engineering, 2008, 28 (6) : 84-89.

[6] Wei-Yong Huang, Tong Min-Ming, Zi-hui Ren. Nonlinear combination forecasting method of gas emission based on SVM [J]. Journal of China University of Mining and Technology, 2009, 38(2): 234-239.

[7] Yi-fei Zhao, Jing-hua Gao. Fault diagnosis based on ant colony optimization neural network [J]. Journal of Beijing Information Science and Technology University, 2010, 25(2):45-48.

[8] Li-xin Zhang, Yu-feng Jiang, Liang Dong. Crack opening ant colony neural network prediction model based on [J]. Hydroelectric power, 2010, 36(5):31-33.

[9] Ren-fa Sheng, Hai-qi Zheng, Hai-wei Jing. Application of MMAS and rough set in fault diagnosis of bearing [J]. Vibration, testing and diagnosis, 2010, 30 (2) : 128-131.

[10]Jing-ming Yang, Xiao-na Sun, Hai-jun Che, etc. Rolling force prediction of cold continuous rolling mill of neural network based on ant colony algorithm [J]. Steel, 2009, 44 (3):52-55. 